\title{
An Efficient Home-Based Risk of Falling Assessment Test Based on Smartphone and Instrumented Insole
}

\author{
Johannes C. Ayena \\ Department of Applied Science \\ University of Quebec at \\ Chicoutimi, Canada
}

\author{
Landry D. Chapwouo T. \\ Department of Mathematics and \\ Computer Science, \\ University of Quebec at \\ Chicoutimi, Canada
}

\author{
Martin J-D. Otis \\ Department of Applied Science \\ UQAC, REPARTI CENTRE \\ Chicoutimi, Canada
}

\author{
Bob-A.J. Menelas \\ Department of Mathematics and \\ Computer Science \\ University of Quebec at \\ Chicoutimi, Canada
}

\begin{abstract}
The aim of this study is to improve and facilitate the methods used to assess risk of falling among older people at home. We propose an automatic version of One-Leg Standing (OLS) test for risk of falling assessment by using a Smartphone and an instrumented insole. For better clinical assessment tests, this study focuses on exploring methods to combine the most important parameters of risk of falling into a single score. Twenty-three volunteers participated in this study for evaluating the effectiveness of the proposed system which includes eleven elderly participants: seven healthy elderly $(67.16 \pm 4.24$ years $)$, four Parkinson disease (PD) subjects $(70 \pm 12.73$ years $)$; and twelve healthy young adults (28.27 \pm 3.74 years). Our work suggests that there is an inverse relationship between OLS score proposed and risk of falling. Proposed instrumented insole and application running on Android could be useful at home as a diagnostic aid tool for analyzing the performance of elderly people in OLS test.
\end{abstract}

Keywords—Falls; OLS test; Elderly; Parkinson's disease.

\section{INTRODUCTION}

Identification of individuals who are at risk of falling due to balance issues is still a major challenge in falls prevention. The risk of falling among elderly persons or persons with neurodegenerative disorder such as Parkinson's disease is generally assessed by clinical tests such as Timed Up and Go test [1], Tinetti test [2] or Berg Balance Scale [3]. Most of these tests are performed in a clinical environment; this could be costly for both time and money for the patient, its family or for the health care system. Falls consequences are even intensified by the demographic change. In near future, there should be more geriatric patients and probably fewer professionals will be available to assess the risk of falling in a clinical environment. As a result, recently, some studies have attempted to bring the risk of falling assessment at home $[4,5]$. These previous research works indicated home-based evaluation has a good potential since it could allow identifying an unstable balance and gait at an early stage. However, the experimental protocol and the devices used are not often straightforward. In addition, clinical values of gait abnormalities identified cannot be interpreted by a layman. Moreover, although many aspects of balance and gait can influence risk of falling, a critical factor is the ability of participant to respond effectively at balance perturbations. These perturbations can arise from the types of ground. For example, soft surfaces can represent an important risk of falling factor [6, 7] and they are not taken into account in the clinical tests assessment at home. Since we evaluate the impact of environmental perturbations, an instrumented One-Leg Standing test is the most appropriate for home-based assessment tools compared to Timed Up and Go or Tinetti tests. The aim of our work is to evaluate the relevance of an inexpensive home-based system for computing a risk of falling and for training an elderly or person with Parkinson disease at maintaining balance. In order to achieve this goal, in this paper, we propose an automatic version of One-Leg Standing (OLS) test over various types of ground designed on a Smartphone and an instrumented insole. The types of ground such as concrete, parquet, sand and gravel are used to be similar to an everyday environment at home and therefore enhance the ability to differentiate level of balance issues.

Following a review of the state of the art in technologies in home-based balance evaluation, we described the primary contribution of this paper, a design of risk of falling assessment based on our instrumented insole and an Android application, such that the proposed score could be associated to a risk of falling. The first evaluation shows encouraging results which are then discussed for usage at home.

\section{RELATED WORK}

First, a brief overview of instrumented test gait and balance disorder assessment is presented. Thereafter, One-Leg Standing (OLS) test used in this study are covered.

\section{A. Technologies for gait and balance disorder analysis}

In the last decade, instrumented tests for gait analysis have been widely used to assess risk of falling by evaluating gait parameter's variation. Several types of shoes equipped with instrumented insoles were developed using various technologies $[8,9]$. Instrumented insole demonstrates the capabilities to compute walking parameters such as pressure located at the heel and toes, and the midstance time [10]. Other studies present an 
instrumented insole able to detect gait phases such as swing time, double support time, stride length and cadence [11]. The instrumented gait analysis system enables planning and assessment of risk of falling among elderly people and is considered as a useful tool for quantifying locomotors performance in people with Parkinson Disease [12, 13]. Zampieri et al [5] studied body-worn sensors for mobility testing at home versus a laboratory testing situation. Their results show that home testing is feasible. Recently, a Smartphone application has proved to be an effective tool for showing clinical tests parameters at home [14]. Other studies showed that a Smartphone-based system may be used for assessing risk of falling [15] and for training users at maintaining balance [16] over different types of ground [17] by using a serious game. These recent studies have shown that mobile systems can be used for assessing clinical test parameters. However, in many recent research works, they usually compare clinical values for differentiating the groups in their works without a formal evaluation with elder participants.

Other studies have been directed toward the development of new instrumented insoles for clinical tests with low-cost and often with wireless communications such as presented in [18, 19]. Those insoles are used for ergonomic evaluation in standing posture and for lower limb prosthetic. Previous studies did not consider the environment of the participant. It is know that the type of ground can affect the gait [7]. Moreover, some studies relate the effects of unstable surfaces such as rocks [20] on the gait parameters. The type of ground becomes a significant factor which should be taken into account in fall risk assessment. Our main contribution in this work is for using such instrumented insole for a home-based system designed in order to assess risk of falling in One-Leg Standing test.

\section{B. One-Leg Standing test}

Many clinical studies showed that the One-Leg Standing (OLS) test is a single task performance and can be used to determine balance issues such as neurological disease, muscular weakness, sensory-motor deficits among elderly [21, 22]. This traditional test also named unipodal test measures the time in seconds (related to a score), which is considered as a level of balance stability. Participants unable to perform this test for at least five (5) seconds are at increased risk of falling. The researchers stated that a time greater than 30 seconds shows a very low risk of falls [22]. However, using only the time in this test is not quite as discriminating for medical decisions. It becomes necessary to measure additional parameters. Indeed during the test, the ability of participant to maintain the strength and weight evenly distributed on the foot is essential for balance control mechanism assessment. The literature has shown that elderly people who present an unstable balance have a greater center of pressure (COP) sway [23]. COP is defined as the point location of the vertical ground reaction, and is often used to identify a balance deficit [24]. The COP displacements in standing posture become a parameter which could be taken into account in assessing risk of falling. Several studies have used a variety of COP measures to predict a risk of falling [25, 26]. They associate postural control capability with balance which is used to describe the body's ability to adjust the center of pressure (COP) near the center of mass (COM). The center of pressure has been commonly used as cues of postural stability in standing. To our best knowledge, no work has attempted to evaluate risk of falling by computing a score over different types of ground with the clinical OLS test by using a Smartphonebased system comprising an instrumented insole.

\section{PROPOSED SYSTEM}

This section presents Smartphone-based system for risk of falling which enable to using One-Leg Standing test at home. We present first our instrumented insole and then a Smartphone application which computes a risk of falling.

\section{A. Instrumented insole}

This apparatus is an intelligent system, recently developed, labelled ACHILE. In the following, ACHILE stands for Active Human-Computer Interface for Locomotion Enhancement. A schematic of the instrumented insole prototype used is shown in Fig.1. It aims at preventing accidental falls related to conditions of the physical environment (slippery ground, steep slope, etc.), or abnormalities of its gait. This device counts a set of sensors such as accelerometers (located in electronic board), force sensors and bending variable sensor whose the location has been showed in [27]. The sensors located inside this insole were selected in order to creating an instrumented system able to sensing many parameters that characterize both gait and balance. All these sensors are exploited to compute the risk level associated to gait risk of falling in standing posture.

To assess force distribution under foot, Force Sensitive Resistors (FSRs) were placed over the insole at strategic pressure position. Two FSRs were placed at the heel, one at right $\left(\mathrm{F}_{\mathrm{hr}}\right)$ and the second at left $\left(\mathrm{F}_{\mathrm{hl}}\right)$. Two others FSRs were placed at the toes, one at left $\left(\mathrm{F}_{\mathrm{t} 1}\right)$ and the second at right $\left(\mathrm{F}_{\mathrm{tr}}\right)$. In sum, this system measures the forces applied at four points under the foot. The force sensors data variation recorded by the smartphone during the OLS test has been used in order to assess the participant's stability.

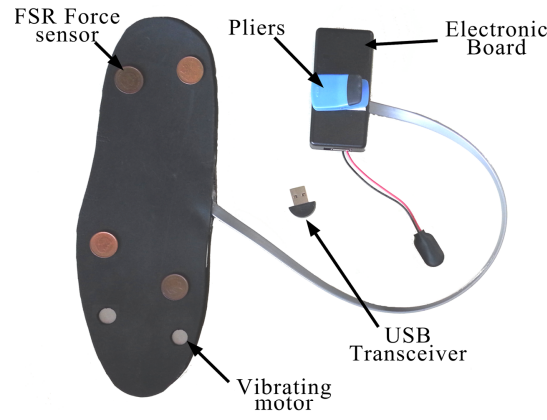

Fig. 1. Instrumented insole used for OLST application. 


\section{B. Android application}

The clinical test implemented in this study, labelled OLST application is designed for an Android operating system. OLST application records raw data signals from four FSR sensors in order to estimate user performance that is doing the test. The software incorporates two main sections which are: 1) instructions on how to properly complete the clinical test, 2) data analysis and results visualization. As the user is standing balanced on one leg, the test could begin by pressing start button. A countdown timer starts from this event, which is the normative time [22] for the user to perform the OLS test. The application stops the test and data are recorded at the end of the normative time required. The daily usage of OLST application allows a remote monitoring of elderly and also could inform about the impact of drugs or rehabilitation interventions on people with Parkinson disease. The login and password of user are registered into data-base in order to showing its progression by comparing performance history and current score with the highest ones performed previously or in last days. The user can make countless tries knowing data are always recorded. For user safety, especially elderly people, and also for smooth running of the test, another person must activate OLST application.

\section{RISK OF FALLING EVALUATION}

In this section, we describe the proposed methodology for computing risk of falling which includes COP parameters computed from four force sensors.

\section{A. COP Positions}

Distributed contact forces acting on the insole surface bounded by the four sensors (shown in Fig.2) may be replaced by a single equivalent force $\left(F_{i}\right)$ and located at position $P_{i}$ (COP's position). The COP displacements on insole surface were defined by the $\mathrm{X}$ and $\mathrm{Y}$ axis. The $P_{i}$ position was then calculated using relations (1) and (2) where $F_{1 z} F_{2 z} F_{3 z} F_{4 z}$ are respectively the scalar of force sensor at location $\mathrm{P}_{j(j=1 \text { to } 4)}$ along Z-axis (perpendicular to insole surface); $a, b$ distances between the force sensors and $F_{i z}$ is the total scalar force. All scalar force sensors are functions of time (t) while $a, b$ are constant values.

$$
\begin{aligned}
& P_{i x}=\left(a / F_{i z}\right) *\left[\left(F_{2 z}+F_{1 z}\right)-\left(F_{4 z}+F_{3 z}\right)\right] \\
& P_{i y}=\left(b / F_{i z}\right) *\left[\left(F_{4 z}+F_{2 z}\right)-\left(F_{1 z}+F_{3 z}\right)\right]
\end{aligned}
$$

\section{B. Proposed OLS score}

The score was calculated following these three steps:

1) The instrumented insole allows measuring the standing time $(T)$ taken by each user. In order to assess the risk of falling by computing a score, we have divided this time by the normative time $\left(T_{N R}\right)$ which depends on age and gender. The normative values for age, gender and eyes in open or closed condition have been shown in [22].

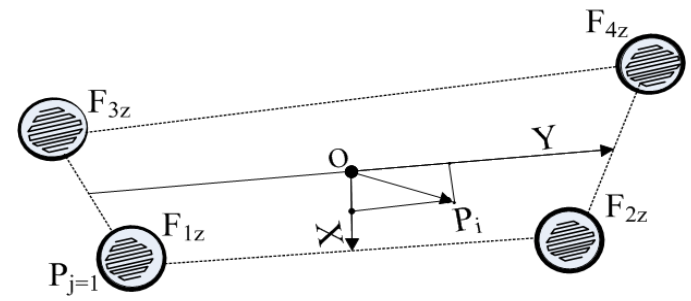

Fig. 2. COP location on insole area in both two directions.

2) $\quad V_{P}$ represents the overall COP velocity [28] during the test and was calculated at each data $(n)$ recorded as follows.

$$
V_{P[n]}=\left[V_{X}^{2}{ }_{[n]}+V_{Y}^{2}{ }_{[n]}^{1 / 2}\right.
$$

The overall analysis of COP movement is preferred compared to a separate analysis of both $x$ and $y$-axis components. The mean of COP velocity $\left(M_{V}\right)$ expressed by (4) is also calculated

$$
M_{V}=(1 / N) * \sum_{n=1}^{N} V_{P[n]}
$$

where $N$ is total data recorded during the test. Indeed, the mean of COP velocity is the most significant parameter that showed, in previous clinical study, a relationship between balance control and quiet standing [29]. Moreover an inverse relationship between OLS score and the mean of COP velocity was demonstrated in [30]. The mean of COP velocity quantifies the neuromuscular activity required to maintain balance. This reflects the postural control of subject, and unstable balance in the OLS test was evaluated using a measure of mean of COP velocity in both anteroposterior and lateral.

3) In this study, we calculate the standard deviations of COP in the two directions. The ratio $(\alpha)$ between these two values, shown in relation (5), is equal to the ratio between the slope of a linear regression $(\mathrm{m})$ and the linear correlation coefficient of Pearson ( $r$ ) for all COP positions on insole surface. In order to improve the assessment of real performance, and to detect a stable position or a balance issue of participant, we have multiplied the parameters showed in previous steps by this ratio $(\alpha)$ which can represent the ability to maintain the strenght and weight even distributed on the foot. The multiplication of parameters described previously provides a result ranging between 0 and 100 corresponding inversely at different risk of falling levels. To maximise this ratio and then the result, the participant must control his balance and reduce his sway along anteroposterior and lateral directions.

$$
\alpha=\left(\sigma_{y} / \sigma_{x}\right)=m / r
$$

We hypothesis that unstable balance can be measured by specific COP parameters combined into a single score; and could be correlated with an increased symptoms of Parkinson disease or others balance problems among elderly. This single score $\left(S_{O L S}\right)$ could be expressed by:

$$
S_{O L S}=\left(T / T_{N R}\right) *\left(1 / M_{V}\right) *\left(\sigma_{y} / \sigma_{x}\right)
$$




\section{Methodology}

Twenty-three (23) volunteers, which includes eleven (11) elderly (7 healthy, 4 PD subjects) and twelve (12) healthy young adults, have involved in this study (Table I). Each PD subject was diagnosed by a movement disorders neurologist at state 2 or 3 . Healthy elderly (subjects without Parkinson disease) were physically independent (University staff and other people outside University) while the healthy young adults were students. The evaluation was carried out in our laboratory. Criteria for inclusion were as follows: elderly subjects were between 59 and 79 years old while young adults were between 22 and 35 years old. All subjects were informed about the experimental protocol of this study and gave written consent before participating. The experience and consent form had been previously approved by the local ethics committee (certificate number 602.434.01). The experiment was performed as follows:

1) In a first time, it is necessary to validate the OLS score suggested in equation (6). To achieve this goal, we performed with young participants, the OLS test over concrete ground at various times between 3 to $T_{N R}$ seconds. This part of the experience has helped us to determine a correlation between suggested score and time in seconds suggested respectively for high or very low risk for OLS test. In a second time, the test was performed at normative time with them over four types of ground such as concrete, gravel, sand and parquet. Two types of ground (concrete and parquet) are grounds encountered most at domestic environment and the others were chosen according to its difficulty to maintain balance.

2) Since older people have difficulty to maintaining their balance, OLS test was performed at normative time with them over concrete. It was asked elderly participants to maintain balance at least five (5) seconds up to the normative time. Each participant in this study was asked to put the instrumented insole in their right shoe and to do the test using OLST application. It was instructed to stand upright on the right foot without support of the upper extremities by looking straight ahead. The OLS test was performed with eyes open condition in all cases above. Overall, the number of seconds a participant was able to maintain a stable position was recorded by the application. The test ended when the stop button is pressed, when the foot up touches the support leg, when the foot up touches the ground or when the arms touch something for improving balance control.

TABLE I. CHARACTERISTICS OF PARTICIPANTS IN THIS STUDY

\begin{tabular}{|c|c|c|c|}
\cline { 2 - 4 } \multicolumn{1}{c|}{} & Healthy Elderly $(\mathrm{n}=7)$ & Young $(\mathrm{n}=12)$ & $\mathrm{PD}(\mathrm{n}=4)$ \\
\hline Age $($ years $)$ & $67.16 \pm 4.24$ & $28.27 \pm 3.74$ & $70 \pm 12.73$ \\
\hline Gender & $4 \mathrm{M} / 3 \mathrm{~F}$ & $10 \mathrm{M} / 2 \mathrm{~F}$ & $3 \mathrm{M} / 1 \mathrm{~F}$ \\
\hline Height $(\mathrm{cm})$ & $165.6 \pm 20.67$ & $174.83 \pm 9.62$ & $162.75 \pm 26.0$ \\
\hline Weight $(\mathrm{Kg})$ & $70.34 \pm 6.59$ & $70.16 \pm 9.94$ & $79.75 \pm 8.26$ \\
\hline
\end{tabular}

During each trial, data from instrumented insole were acquired and wirelessly transmitted via Bluetooth in real time to Smartphone. The tests were carried out preferably in the morning so that the participant should be in good performance and mental state (ensuring tiredness does not alter results). After OLS test, each participant was also asked to walk three meters over each type of ground. Then, the participant describes how he felt and if he struggled with disturbances or with obstacle on the ground. He determines the ground on which it is easier or difficult to walk and the risk of falling level according to Likert scale (scale from 1 to 5 ).

\section{RESULTS}

The relation between balance control and data from force sensors are computed by the Smartphone application into single score. The scores obtained with young participants have been divided into four groups corresponding to the four types of ground. One way Analysis of Variance (ANOVA) was performed in order to compare level of stability by using the scores over concrete, parquet, sand and gravel. The ANOVA results is reported as an F-statistic with its associated degrees of freedom (3 and 44) and p-value. The null hypothesis $H_{0}$ is that all the means of score from different ground are equals. Given that the null hypothesis is rejected if $F>F_{\text {critical }}$ at the 0.05 level of significiance, the computed test statistic $F$ in our work is 20.89, which is more than $F_{\text {critical }}=2.82$. This analysis of variance, in Fig. 3, leads to the conclusion that there has a significant effect related to the types of ground and risk of falling $\left(p=1.44 * 10^{-8}<0.05\right)$. However, comparing only concrete and parquet, no significant effect was observed $(F=0.19<4.3)$. Table II reports p-values of parquet, sand and gravel compared with concrete. Higher severity of balance disorder was associated with lower OLS scores, which indicated more mobility deficits (as shown in Fig.4). Results from simple questions to participants have shown that none of them included in this study has proven difficult to walk on concrete or parquet (level<2, Fig.5). Similarly, any perceived imbalance has been notified on these two floors $(0 \%) .41 .6 \%$ of young participants, $71.43 \%$ of healthy elderly and all of PD subjects have been notified to perceived imbalance and difficulty to walk over sand. $25 \%$ of PD subjects perceived imbalance and difficulty to walk over gravel. The risk of falling remains higher over sand and gravel according to Likert scale using all participant's responses especially for elderly people.

TABLE II. P-VALUES :TYPES OF GROUND COMPARED WITH CONCRETE

\begin{tabular}{|c|c|c|}
\hline Concrete-Parquet & Concrete-Sand & Concrete-Gravel \\
\hline $0.66^{\mathrm{a}}$ & $4.3 * 10^{-5}$ & $3.46 * 10^{-6}$ \\
\hline
\end{tabular}

${ }^{\mathrm{a}}$ Indicates p-value more than 0.05 


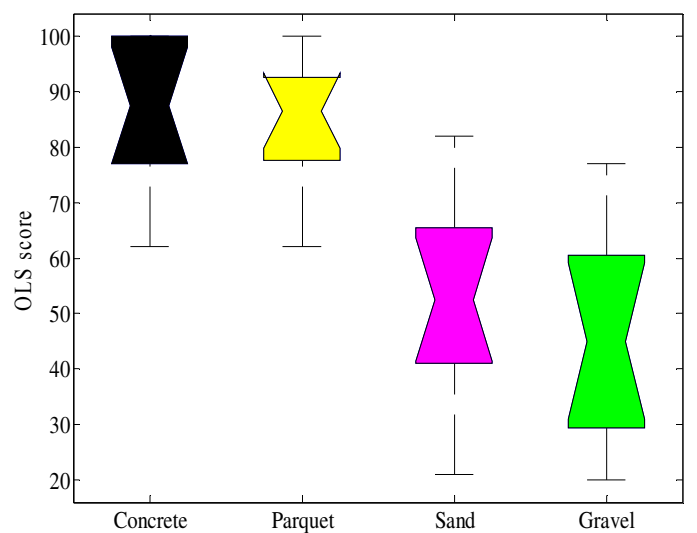

Fig. 3. Anova analysis for OLS's score over four types of ground at normative value $T_{N R}$ associate with age and gender of young participants $\left(p=1.44 * 10^{-8}<\right.$ $0.05)$.

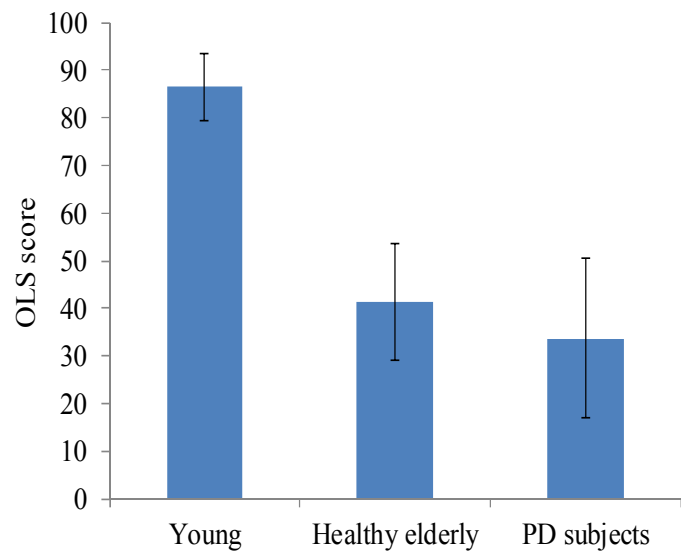

Fig. 4. Score level computed by the OLST application for all participants over concrete (mean and vertical bars are standard errors).

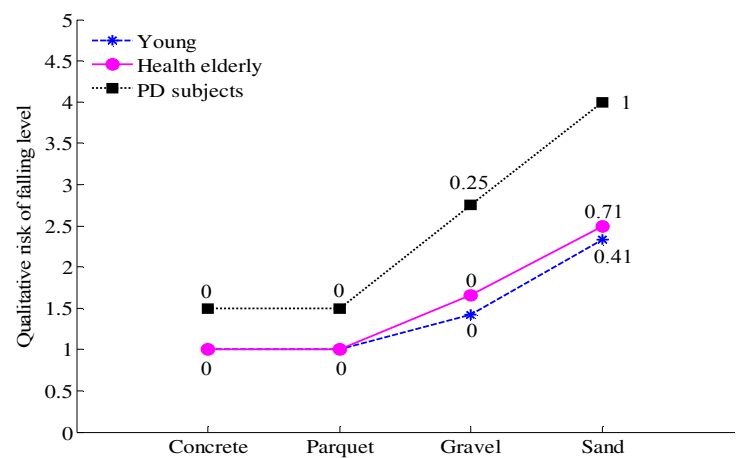

Fig. 5. Results questionnaires: 1) Walk over types of ground (from easiest to most difficult) and the average risk of falling according to Likert scale; 2) The number of participants who felt an imbalance (numbers showed as a ratio).

\section{DISCUSSION}

The main purpose of this study was to improve the methods used for evaluating balance control in elderly at home especially people with Parkinson disease. In our study, we determined the relationship between a score and a risk of falling by comparing three participants groups and evaluating the effect of the types of ground. It was observed that the type of ground did affected OLS score (Fig.3). The risk of falling depends therefore on type of ground (ground properties such as compliance and coefficient of friction). By using concrete ground as reference, the OLS score over concrete and sand or over concrete and gravel is statistically significant (Table II). This difference is more pronounced over gravel: aggregate size of this material is bigger than sand, which has probably causes some body sway and foot motion more often. However, the risk of falling according to Likert scale is more pronounced with sand (Fig.5) which could be due to difficulty to walk over sand. No significant difference was found between concrete and parquet $(p=0.66>0.05)$. This could be explained by the fact that both types of ground are rigid (less foot motion and body sway). It was noticed by elderly balance control is different over each type of ground (Fig.5), which means environment is an important factor in risk of falling. Most of elderly participants have difficulty to maintain balance until the normative value. Among the elderly, the left foot touches regularly the ground or support during the OLS test before the normative value required except three healthy elderly and one PD subject.

\section{VIII.CONCLUSION}

A novel methodology approach to risk of falling evaluation at home has been designed. Evaluation with participants (healthy young, healthy elderly and Parkinson disease subjects) demonstrates the feasibility of our approach. We have implemented an automatic system providing the risk of falling based on a score level. As suggested by our results, COP parameters analyzed during OLS test seem to be a useful for evaluating risk of falling at home among elderly. The score level computed by OLST application can be also used as a motivation in order to improve the physical condition of elderly. Many tests were conducted and our findings suggested that the proposed score could be a good candidate, which could possibly help us to evaluate and train balance among elderly and people with Parkinson disease in the near future at home. In this paper, we also analyzed the ground's effect on balance parameters of human by analyzing its different positions in two directions (anteroposterior and lateral). The type of ground increases the imbalance and can lead a walker to fall. The different types of the ground that an elderly might walk on which must be a hint for future works to pay attention to this issue. Future works which includes some others perturbations will be conducted at home for further of the proposed system.

\section{ACKNOWLEDGMENTS}

The authors would like to thank Valere Plantevin for helpful ideas implementation of this application. We also wish to thank Dr. Louis Tremblay, physiotherapist attached to the Parkinson Society of Saguenay, for assistance. 


\section{REFERENCES}

[1] G. Thrane, R. Joakimsen, and E. Thornquist, "The association between timed up and go test and history of falls: The Tromso study," BMC Geriatrics, vol. 7, p. 1, 2007.

[2] M. E. Tinetti, D. I. Baker, G. Mc Avay, E. B. Claus, P. Garrett, M. Gottschalk, et al., "A multifactorial intervention to reduce the risk of falling among elderly people living in the community," N Engl J Med, vol. 331, pp. 821-827, 1994.

[3] S. W. Muir, K. Berg, B. Chesworth, and M. Speechley, "Use of the Berg Balance Scale for predicting multiple falls in community-dwelling elderly people: a prospective study.," Phys Ther, vol. 88(4), pp. 449-59, 2008 Apr.

[4] T. Frenken, M. Brell, M. Gövercin, S. Wegel, and A. Hein, "aTUG: technical apparatus for gait and balance analysis within component-based Timed Up \& Go using mutual ambient sensors," Journal of Ambient Intelligence and Humanized Computing, vol. 4, pp. 759-778, 2013.

[5] Z. Cris, S. Arash, C.-K. Patricia, G. John, and B. H. Fay, "Assessing mobility at home in people with early Parkinson's disease using an instrumented Timed Up and Go test," Parkinsonism and Related Disorders vol. 17 pp. 277-280, 2011.

[6] S. Sprager and D. Zazula, "Impact of different walking surfaces on gait identification based on higher-order statistics of accelerometer data," in Signal and Image Processing Applications (ICSIPA), IEEE International Conference on, 2011, pp. 360-365.

[7] D. S. Marigold and A. E. Patla, "Adapting locomotion to different surface compliances: neuromuscular responses and changes in movement dynamics," Journal of neurophysiology, vol. 94, pp. 1733-1750, 09/ 2005.

[8] S. J. M. Bamberg, A. Y. Benbasat, D. M. Scarborough, D. E. Krebs, and J. A. Paradiso, "Gait Analysis Using a Shoe-Integrated Wireless Sensor System," Information Technology in Biomedicine, IEEE Transactions on, vol. 12, pp. 413-423, 2008.

[9] C. Meng, H. Bufu, and X. Yangsheng, "Intelligent shoes for abnormal gait detection," in Robotics and Automation, IEEE International Conference on, 2008, pp. 2019-2024.

[10] F. Martinez-Marti, M. S. Martinez-Garcia, S. G. Garcia-Diaz, J. GarciaJiménez, A. J. Palmo, and M. A. Carvajal, "Embedded sensor insole for wireless measurement of gait parameters," Australian Physical \& Engineering Sciences in Medecine, vol. 37, pp. 25-35, March 2014.

[11] H. Noshadi, S. Ahmadian, H. Hagopian, J. Woodbridge, F. Dabiri, N. Amini, et al., "Hermes: Mobile balance and instability assessment system," In Conference on Bio-inpsired Systems and Signal, BIOSIGNALS, pp. 264-270, Jan 2010.

[12] W. Aner, H. Talia, G. Nir, and M. H. Jeffrey, "Objective Assessment of Fall Risk in Parkinson's Disease Using a Body-Fixed Sensor Worn for 3 Days," PLoS ONE, vol. 9, 2014.

[13] A. S. Buchman, S. E. Leurgans, A. Weiss, V. VanderHorst, A. Mirelman, R. Dawe, et al., "Associations between quantitative mobility measures derived from components of conventional mobility testing and Parkinson gait in older adults," PLoS One, vol. 9(1), Jan 2014.

[14] M. Milosevic, E. Jovanov, and A. Milenkovic, "Quantifying Timed-Upand-Go test: A smartphone implementation," in Body Sensor Networks (BSN), IEEE International Conference on, 2013, pp. 1-6.

[15] S. Brassard, M. J.-D. Otis, A. Poirier, and B.-A. J. Menelas, "Towards an automatic version of the Berg balance scale test through a serious game," presented at the Proceedings of the Second ACM Workshop on Mobile Systems, Applications, and Services for HealthCare, Toronto, Ontario, Canada, 2012.
[16] D. Meldrum, A. Glennon, S. Herdman, D. Murray, and R. McConn-Walsh, "Virtual reality rehabilitation of balance: assessment of the usability of the Nintendo Wii ${ }^{\circledR}$ Fit Plus," Disability and Rehabilitation: Assistive Technology, vol. 7, pp. 205-210, 2012.

[17] B.-A. J. Menelas and M. J. D. Otis, "A Serious Game for Training Balance Control over Different Types of Soil," in Serious Games Development and Applications. vol. 7528, M. Ma, M. Oliveira, J. Hauge, H. Duin, and K.-D. Thoben, Eds., ed: Springer Berlin Heidelberg, 2012, pp. 31-42.

[18] M. Stalin and C. L. Bennett, "Development of an Insole System for Realtime Capture of Ground Reaction Forces in Lower-limb Amputees," in Biomedical Engineering Conference (SBEC), 29th Southern, May 2013, pp. 137-138.

[19] A. Cristiani, G. M. Bertolotti, E. Marenzi, and S. Ramat, "An instrumented insole for long term monitoring movement, comfort and ergonomics," IEEE Sensors Journal, vol. 14(5), May 2014.

[20] D. H. Gates, J. M. Wilken, S. J. Scott, E. H. Sinitski, and J. B. Dingwell, "Kinematic Strategies for Walking Across a Destabilizing Rock Surface()," Gait \& posture, vol. 35, pp. 36-42, 09/03 2012.

[21] T. Michikawa, Y. Nishiwaki, T. Takebayashi, and Y. Toyama, "One-leg standing test for elderly populations," Journal of Orthopaedic Science, vol. 14, pp. 675-685, 2009.

[22] B. A. Springer, R. Marin, T. Cyhan, H. Roberts, and N. Gill, "Normative values for the unipedal stance test with eyes open and closed," J Geriatr Phys Ther, vol. 30(1), pp. 8-15, 2007.

[23] F. Menegoni, E. Tacchini, M. Bigoni, L. Vismara, L. Priano, M. Galli, et al., "Mechanisms underlying center of pressure displacements in obese subjects during quiet stance," Journal of NeuroEngineering and Rehabilitation, vol. 8, p. 20, 2011.

[24] A. W. O. Gil, M. R. Oliveira, V. A. Coelho, C. E. Carvalho, D. C. Teixeira, and R. A. d. Silva Jr, "Relationship between force platform and two functional tests for measuring balance in the elderly," Brazilian Journal of Physical Therapy, vol. 15, pp. 429-435, 2011.

[25] M. Piirtola and P. Era, "Force platform measurements as predictors of falls among older people - a review," Gerontology, vol. 52(1), pp. 1-16, 2006.

[26] M. Moghadam, H. Ashayeri, M. Salavati, J. Sarafzadeh, K. D. Taghipoor, A. Saeedi, et al., "Reliability of center of pressure measures of postural stability in healthy older adults: Effects of postural task difficulty and cognitive load," Gait \& Posture, vol. 33, pp. 651-655, 4// 2011.

[27] B.-A. J. Menelas and M. J.-D. Otis, "Toward an Automatic System for Training Balance Control Over Different Types of Soil," in Virtual, Augmented Reality and Serious Games for Healthcare 1. vol. 68, M. Ma, L. C. Jain, and P. Anderson, Eds., ed: Springer Berlin Heidelberg, 2014, pp. 391-408.

[28] L. J. Smart, T. A. Stoffregen, and B. G. Bardy, "Visually induced motion sickness predicted by postural instability," Hum. Fact, vol. 44, pp. 451465, 2002.

[29] R. Stemplewski, J. Maciaszek, W. Osiński, and R. Szeklicki, "Test-Retest Reliability of Measurements of the Center of Pressure Displacement in Quiet Standing and During Maximal Voluntary Body Leaning Among Healthy Elderly Men," J Hum Kinet, vol. 28, pp. 15-23, Jun 2011.

[30] A. G. Rodrigo, S. Rony, A. R. Francisco, A. A. David, and A. O. Pablo, "Relationship between unipedal stance test score and center of pressure velocity in elderly," Rev Esp Geriatr Gerontol, vol. 46(5), pp. 256-60, 2011 Sep-Oct. 